\title{
A mortalidade entre adolescentes egressos do sistema penal
}

\author{
Mortality among former youth offenders
}

Vinícius Mauat da Silva (https://orcid.org/0000-0001-8859-6440) ${ }^{1}$

Pedro do Valle Teichmann (https://orcid.org/0000-0003-0617-0927) ${ }^{1}$

Thomas Scanlon (https://orcid.org/0000-0002-9727-2795) ${ }^{2}$

José Vicente Tavares dos Santos (https://orcid.org/0000-0001-8410-5085) ${ }^{3}$

Marcelo Zubaran Goldani (https://orcid.org/0000-0002-5302-284X) ${ }^{1}$
${ }^{1}$ Núcleo de Estudos da Saúde da Criança e do Adolescente, Hospital de Clínicas de Porto Alegre. R. Ramiro Barcelos 2350, Santana. 90035-903 Porto Alegre RS Brasil. vmauat@gmail.com ${ }^{2}$ Center for International Child Health, Institute of Child Health, University College London. Londres Inglaterra.

${ }^{3}$ Departamento de PósGraduação em Sociologia e em Políticas Públicas, Universidade Federal do Rio Grande do Sul. Porto Alegre RS Brasil.

\begin{abstract}
The objective of this article is to analyze the detention of youth offenders involved in the juvenile justice system in the State of Rio Grande do Sul (FASE-RS), the reason for detention, and mortality among former young offenders. We conducted an observational study with youth offenders discharged from facilities run by FASERS in Porto Alegre between 2002 and $2012(n=$ 8,290). We collected the following information: date of discharge, offence committed, skin color, gender, and duration of detention. The data was crosschecked with data from the state's Mortality Information System to identify deaths among former young offenders up to December 2014. The predominant offences were crimes against property and drug-related crimes. The large majority of youth detained for drug-related offences were admitted for offences related to drug trafficking. There was a seven-fold increase in drug-related offences over the period. Death was associated $(p<0.001)$ with being male and number of reentries (>3). The sample's mortality rate was high and the main cause of death was homicide. The findings suggest that young offenders face high levels of psychosocial vulnerability. There was an association between minor crimes and high rates of mortality among former young offenders.

Key words Youth, Institutionalized, Detainees, Mortality, Homicide
\end{abstract}

Resumo $O$ objetivo deste artigo é analisar as causas de internações da FASE do Estado do Rio Grande do Sul e a sua relação com a mortalidade de adolescentes egressos entre os anos de 2002 a 2014. Estudo observacional realizado com o banco de dados de adolescentes privados de liberdade nas unidades da FASE-RS de Porto Alegre, desligados nos anos de 2002 a $2012(n=8290)$. Informações sobre data de desligamento, ato infracional, tempo de internação e variáveis biológicas foram obtidas de banco de dados. Essa amostra foi comparada com o Sistema de Verificação de Óbitos da Secretaria Estadual de Saúde para analisar a mortalidade entre os anos de 2002 e 2014. Os adolescentes foram internados predominantemente por atos infracionais de cunho patrimonial e ligados a entorpecentes, os quais sofreram um aumento de aproximadamente $700 \%$ no periodo. O desfecho óbito associou-se $(p<0,001)$ às variáveis gênero masculino e número de entradas ( $\geq 3$ ). Essa amostra apresentou alta taxa de mortalidade sendo a principal causa homicídio. Os achados evidenciam o alto grau de vulnerabilidade psicossocial dos egressos do sistema penal juvenil de internação. Nota-se uma associação entre crimes de baixo poder ofensivo e altas taxas de mortalidade pós-liberdade.

Palavras-chave Adolescente institucionalizado, Prisioneiros, Mortalidade, Homicídio 


\section{Introdução}

O Brasil apresenta alta taxa de mortalidade entre adolescentes, a qual relaciona-se com diferentes classes sociais de forma assimétrica, condicionada por fatores sociais, econômicos, culturais, ambientais e subjetivos. Neste caso, indivíduos e comunidades são desigualmente expostos a fatores de proteção e, como reflexo, assimetricamente submetidos a risco de mortalidade.

O código penal brasileiro considera todo o indivíduo menor de 18 no momento da ação ou omissão como inimputável, ou seja, não será responsabilizado penalmente por seus atos, porém sua conduta será apurada por um estatuto próprio, o Estatuto da Criança e do Adolescente (ECA). O ECA define como o adolescente em conflito com a lei é responsabilizado por seus atos, considerando-se a sua condição de pessoa em desenvolvimento e possuidor de todas as garantias processuais e penais ${ }^{1}$.

A definição de criança e adolescente está contida em seu art. $2^{\circ}$ : “Considera-se criança, para os efeitos desta Lei, a pessoa até doze anos de idade incompletos, e adolescente aquela entre doze e dezoito anos de idade". Logo, todos aqueles entre 12 anos completos e 18 anos incompletos de idade, e autores de infrações penais, terão sua conduta regulada pelo texto proposto pelo ECA, através da aplicação de medidas socioeducativas ${ }^{1}$. No Rio Grande do sul, a Fundação de Atendimento Socioeducativo (FASE-RS), foi criada em 2002 e passou a responsabilizar-se pela execução de medidas socioeducativas, de semiliberdade e internação de adolescentes autores de ato infracional, sendo considerada a permanência em medida privativa de liberdade em 3 anos no máximo, ou até o adolescente completar 21 anos. O atendimento prestado na FASE-RS é norteado pelo Programa de Execução de Medidas Socioeducativas de Internação e Semiliberdade (PEMSEIS), sustentado pelo eixo principal advindo do Plano Individual de Atendimento (PIA). Em janeiro de 2012, a Lei $n^{\circ} 12.594$ foi sancionada, instituindo o Sistema Nacional de Atendimento Socioeducativo (SINASE), o qual uniformizou, em todo o território nacional, o atendimento aos adolescentes em conflito com a lei e o processo de apuração das infrações cometidas ${ }^{2,3}$.

De um modo geral, o adolescente interno no sistema penal juvenil é da etnia negra, provem de classe social vulnerável, com ensino fundamental incompleto, não frequentam a escola e não estão inseridos no mercado de trabalho ${ }^{4}$. O Mapa da Violência de 2016 explicita a participação dos jovens (15 a 29 anos) no contexto da violência brasileira, visto que esses representaram 58\% das vítimas de homicídio por arma de fogo no ano de 2016, enquanto, segundo o IBGE, compunham apenas $26 \%$ da população total no período ${ }^{5}$. Apesar da relevância do tema, na literatura ligada ao campo da saúde, há poucos estudos que abordem especificamente a questão da mortalidade neste grupo de adolescentes, considerando sua alta vulnerabilidade e a complexidade de aspectos sociodemográficos envolvidos. Portanto, este estudo se propõe a analisar a internação em unidade socioeducativa, o seu motivo e o desfecho mortalidade entre adolescentes egressos, considerando as unidades da FASE-RS. Sua importância está ligada ao ineditismo deste levantamento e ainda à notoriedade que o tema vem ganhando na mídia nacional, gerando discussões acerca da recuperação de adolescentes envolvidos em atos infracionais, bem como a redução da maioridade penal. Mais que a simples exposição descritiva, este trabalho busca lançar um olhar inédito e multidisciplinar sobre os adolescentes privados de liberdade, contribuindo para a ampliação da compreensão desse fenômeno.

\section{Métodos}

Estudo exploratório observacional descritivo em adolescentes em conflito com a lei egressos das unidades da Fundação de Atendimento Socioeducativo de Porto Alegre (FASE-RS), entre $1^{\circ}$ de janeiro de 2002 e 31 de dezembro de 2012. O corte correspondeu à última data de desligamento dos indivíduos das unidades. No caso de reentrada(s) na instituição, foi adotado como critério o último desligamento.

Foram incluídos no estudo adolescentes em cumprimento de medida socioeducativa e aqueles provisoriamente internados antes de a sentença ser prolatada pelo prazo máximo de 45 dias, em qualquer uma das unidades de Porto Alegre (RS). Excluiu-se adolescentes que não indicavam filiação materna elou data de nascimento, ou ainda aqueles que as indicavam de forma incompleta.

As informações foram extraídas do banco de dados da Assessoria de Informação e Gestão (AIG) da FASE/RS, cuja guarda e armazenamento é realizado pela Companhia de Processamento de dados do Estado do Rio Grande do Sul (PROCERGS).

Foi realizado o cruzamento entre o nome completo do adolescente e as informações do Sis- 


\section{Variáveis de interesse}

Ato Infracional: categorizada com base no bem jurídico tutelado, ou seja, bens eleitos pelo código penal como merecedores de proteção ${ }^{6}$. Foram elencadas quatro categorias:

- Contra a pessoa: inclui crimes contra a vida, lesões corporais, periclitação da vida e da saúde, rixa, crimes contra a honra e crimes contra a liberdade individual.

- Contra o patrimônio: inclui furto, roubo e extorsão, usurpação, dano, apropriação indébita, estelionato e outras fraudes e receptação.

- Entorpecentes: inclui os atos infracionais análogos aos crimes relacionados com entorpecentes, quer seja voltado ao comércio ou para consumo próprio, previstas na lei 11.343\06, atualmente em vigor, ou ainda previstas na legislação da época. Nestes casos, a doutrina aponta que o bem jurídico tutelado é a saúde pública, visto que o consumo de psicoativos prejudicaria a saúde de seus usuários?.

- Outros motivos de internação: inclui atos inftacionais que não se enquadram nas categorias acima, como atentado violento ao pudor e estupro, além de internações "não-relacionadas à ato infracional": retorno espontâneo à unidade, descumprimento de medida anteriormente imposta, apresentação fora do prazo (quando do retorno de atividades externas) apresentação de outras comarcas, evasão de abrigo.

Causa do óbito: foi categorizada nos seguintes itens, excludentes entre si:

- Homicídios: inclui todas as agressões intencionais infligidas por outra pessoa, através de qualquer meio, cujo resultado tenha sido o óbito. Estão incluídos nesta categoria os óbitos decorrentes de intervenções legais (provocadas por agentes de Estado no cumprimento do seu dever).

- Suicídios: inclui lesões ou envenenamento autoprovocadas intencionalmente.

- Outras causas: inclui causas de óbito que não se incluíram em nenhuma das categorias acima, como, por exemplo, afogamento, doença e acidente de transporte.

As variáveis demográficas utilizadas foram: cor da pele (branco/não branco), gênero (masculino/feminino) e idade na internação em anos (considerando a primeira internação, em caso de reentrada).

As variáveis processuais utilizadas foram: motivo da internação (conforme o código penal) ${ }^{6}$, tempo total de internação em anos e número de entradas \reentradas. O tempo de internação foi indicado em seu total, podendo superar os 3 anos (1.096 dias) estabelecidos no ECA, pois o banco de dados utilizado não individualiza o tempo de sucessivos reingressos.

$\mathrm{Na}$ análise estatística, as variáveis quantitativas foram descritas em medianas e valores mínimo e máximo, caso não se tratar de uma distribuição paramétrica. A descrição dos dados foi realizada por meio de frequências absolutas (n) e relativas (\%). O teste de qui-quadrado foi utilizado para a diferença entre as proporções. $\mathrm{O}$ desfecho óbito foi relacionado a variáveis demográficas e processuais categóricas através do teste de qui-quadrado. Esta pesquisa foi aprovada pelo Comitê de Ética em Pesquisa (CEP) do Hospital de Clínicas de Porto Alegre.

\section{Resultados}

Foram submetidos à medida de internação nas unidades da FASE-RS de Porto Alegre, entre janeiro de 2002 e dezembro de 2012, 8.365 adolescentes. Destes, $75(0,89 \%)$ foram excluídos em virtude da inexistência de informações sobre a data de nascimento elou filiação materna, restando 8.290 egressos no estudo. Os adolescentes participantes eram predominantemente brancos, do gênero masculino e tinham idade média de 17,1 anos, variando de 12 a 21 anos A mediana de internação total foi de 48 dias, variando de 1 a 1.832 dias, sendo que 4.000 (48,3\%) dos jovens permaneceram internados por menos de de 45 dias (Tabela 1).

Nota-se, no período de estudo, um aumento significativo de internações motivadas por entorpecentes, as quais passaram de $3 \%$ para $49,4 \%$. Dentro desta categoria, 1.257 adolescentes foram internados por ato infracional análogo ao tráfico de drogas (Figura 1).

Em relação ao desfecho óbito, foram localizados 784 adolescentes egressos deste período com óbito registrado até dezembro de 2014, correspondendo a uma taxa de mortalidade dos egressos de $9,45 \%$. A frequência de óbitos foi maior entre o gênero masculino, nos adolescentes com 
Tabela 1. Relação entre covariáveis demográficas e processuais e óbito de egressos das unidades de internação da FASE de Porto Alegre, 2002-2012. $(\mathrm{n}=8290)$.

\begin{tabular}{|c|c|c|c|c|c|}
\hline & \multirow{2}{*}{ n (8290) } & \multirow{2}{*}{$\%$} & \multicolumn{3}{|c|}{ Óbito (784) } \\
\hline & & & $\mathbf{n}$ & $\%$ & $\mathbf{P}$ \\
\hline Cor da pele & & & & & 0,832 \\
\hline Branca & 4368 & 53,7 & 413 & 9,5 & \\
\hline Não Branca & 3763 & 46,3 & 361 & 9,6 & \\
\hline Não informado & 159 & 1,9 & & & \\
\hline Gênero & & & & & $<0,001$ \\
\hline Masculino & 7490 & 90,3 & 756 & $10,1^{\star}$ & \\
\hline Feminino & 800 & 9,7 & 28 & 3,5 & \\
\hline Motivo da Internação & & & & & $<0,001$ \\
\hline Contra a pessoa & 522 & 8,7 & 45 & 8,6 & \\
\hline Contra o patrimônio & 2776 & 46,5 & 249 & 9 & \\
\hline Entorpecentes & 1299 & 21,7 & 98 & $7,5^{\star *}$ & \\
\hline Outros motivos de internação & 1378 & 23 & 172 & $20,4^{*}$ & \\
\hline Não Informado & 2315 & 27,9 & & & \\
\hline Tempo total de internação (dias) & & & & & $<0,001$ \\
\hline$<45$ & 4000 & 48,3 & 323 & $8,1^{* *}$ & \\
\hline $45-180$ & 1467 & 17,7 & 147 & 10 & \\
\hline $180-360$ & 826 & 10 & 97 & 11,7 & \\
\hline $360-720$ & 1370 & 16,5 & 145 & 10,6 & \\
\hline$>720$ & 627 & 16,5 & 72 & 11,5 & \\
\hline Idade de internação & & & & & 0,056 \\
\hline $12-15$ anos & 727 & 8,8 & 51 & 7 & \\
\hline 15-18anos & 6042 & 72,9 & 581 & 9,6 & \\
\hline $18-21$ anos & 1521 & 18,3 & 152 & 10 & \\
\hline Internações & & & & & $<0,001$ \\
\hline 1 entrada & 4861 & 58,6 & 392 & $8,1^{\star *}$ & \\
\hline 2 entradas & 1810 & 21,8 & 184 & 10,2 & \\
\hline$\geq 3$ entradas & 1619 & 19,5 & 208 & $12,8^{*}$ & \\
\hline
\end{tabular}

maior tempo total de internação e com maior número de entradas no sistema. Nota-se uma menor frequência de óbitos entre adolescentes internados por tráfico. Observa-se o homicídio como a principal causa de óbito entre os adolescentes e também a alta frequência de suicídio entre os egressos. Contudo, não há associação entre causa de óbito e causa de internação (Tabela 2).

\section{Discussão}

Este estudo exploratório abordou um tema pouco discutido no campo da saúde, o qual se tornou um problema de saúde pública negligenciado pela comunidade científica brasileira. Como re- sultado, ficou evidente o alto grau de vulnerabilidade desse grupo de adolescentes, associado a uma alta taxa de mortalidade, mesmo relacionada a infrações de baixo poder ofensivo.

Ao analisar as causas das internações de adolescentes na FASE-RS, observou-se que os atos infracionais análogos aos crimes contra as pessoas se mantiveram estáveis, passando de $7 \%$ de todos os casos, em 2002, para 6,4\% em 2012. Em relação aos demais motivos de internação, houve uma inversão: aqueles relacionados ao patrimônio caíram de $47 \%$ para $32,5 \%$ no final do período. Enquanto que internações motivadas pelo envolvimento com entorpecentes eram 3\% em 2002 e passaram para 49,4\% em 2012, demonstrando que a atual lei e política relacionada ao 
Figura 1. Motivo da internação nas unidades de internação da FASE de Porto Alegre, 2002-2012.

Fonte: SIM/NIS/DGTI/SES/RS.

Tabela 2. Relação entre motivo da internação e causa do óbito dos egressos das unidades de internação da FASE de Porto Alegre, 2002-2012. $(\mathrm{n}=784)$

\begin{tabular}{lcccccc}
\hline & $\begin{array}{c}\text { Contra a } \\
\text { pessoa } \\
(\mathbf{n}=\mathbf{5 2 2})\end{array}$ & $\begin{array}{c}\text { Contra o } \\
\text { patrimônio } \\
(\mathbf{n}=\mathbf{2 7 7 6})\end{array}$ & $\begin{array}{c}\text { Entorpecentes } \\
(\mathbf{n}=\mathbf{1 2 9 9})\end{array}$ & $\begin{array}{c}\text { Outros } \\
\text { motivos de } \\
\text { internação } \\
(\mathbf{n}=\mathbf{1 3 7 8})\end{array}$ & $\begin{array}{c}\text { Não } \\
\text { informado } \\
(\mathbf{n}=\mathbf{2 3 1 5})\end{array}$ & $\begin{array}{c}\text { Total } \\
(\mathbf{n}=\mathbf{8 2 9 0})\end{array}$ \\
\hline Homicídios & $40(7,66)$ & $209(7,52)$ & $89(6,85)$ & $133(9,65)$ & $185(7,99)$ & $656(7,91)$ \\
Suicídio & $2(0,98)$ & $5(0,18)$ & 0 & $9(0,65)$ & $3(0,13)$ & $19(0,23)$ \\
Outras causas de óbito & $3(0,57)$ & $37(1,33)$ & $9(0,69)$ & $28(2,03)$ & $32(1,38)$ & $109(1,31)$ \\
Total & $45(8,62)$ & $251(9,04)$ & $98(7,54)$ & $170(12,33)$ & $220(9,50)$ & $784(9,45)$ \\
\hline
\end{tabular}

Fonte: SIM/NIS/DGTI/SES/RS.

tráfico e consumo de drogas ilícitas intensificou a internação dos adolescentes, principalmente dos advindos de estratos sociais mais vulneráveis.

A hiperinternação motivada pelas drogas não é um fenômeno isolado. Desde 2006, início da vigência da nova lei de drogas, as internações de adolescentes relacionadas às drogas passaram de $6,7 \%$ para $12,6 \%$, entre 2006 e 2007 , e nos anos posteriores se elevaram até chegar a 49,4\%. Uma das hipóteses é a entrada em vigor da lei $11.343 \backslash 2006^{7}$, a chamada "Nova Lei de Drogas", em um contexto internacional de "redução de danos", adotando uma abordagem médico-criminal, na qual o usuário não é privado de liberdade, mas sim encaminhado para o sistema de saúde, enquanto que o traficante sofre penalidade mais rígida ${ }^{8}$.

Percebe-se que a "Nova Lei de Drogas" falhou e perpetuou um caráter altamente punitivista nas políticas antidrogas brasileiras, pois não estabelece de forma clara a distinção entre usuário e traficante. Neste caso, o rótulo de traficante ou 
usuário pode ser influenciado por características sociobiológicas, de modo a poder reproduzir e ampliar as desigualdades diante dos processos de controle. O comportamento criminoso passa a ser visto como uma característica de cidadãos oriundos de estratos menos favorecidos e marginalizados, identificando-os como pertencentes a "classes perigosas". A imagem do traficante é produzida através de um processo de discriminação e medo difusos, que acaba por aproximar traficantes de não traficantes?.

O cenário do tráfico de drogas é bastante violento, tanto em virtude de disputas territoriais entre grupos quanto pela repressão policial. Assim, em um cenário cercado pelo conflito, seria esperado que adolescentes envolvidos com o tráfico de drogas apresentassem um alto índice de óbitos em relação aos demais atos infracionais. Não tendo sido encontrada esta relação, a alternativa indica que estes adolescentes poderiam ser usuários, logo não estariam inseridos na sistemática da violência do comércio de drogas. Além disso, o consumo de drogas, ilícita ou não, já foi apontado como um fator de risco para que o adolescente seja induzido a práticas violentas, visto que prejudica as relações interpessoais, a adoção de uma rotina e a prática de atividades que poderiam melhorar sua saúde e desempenho escolar, o que prejudica a reinserção do adolescente em conflito com a lei à sociedade ${ }^{10}$.

Os homicídios possuem uma padronização jurídica universal, funcionando como um índice para mensurar a intensidade da violência em um determinado espaço social e o risco desse desfecho para a população ${ }^{11}$. O acúmulo de carências, a escassez de fatores de proteção e o baixo acesso a direitos como educação, saúde e moradia também já foram associados aos homicídios de jovens ${ }^{12}$. A falta de rede de apoio também é um fator que predispõe o adolescente a um estilo de vida de alto risco social e pode explicar o seu comportamento transgressor ${ }^{13,14}$. Arruda da Silva et al. ${ }^{15}$ observaram uma amostra de 800 jovens internados em instituto de proteção em que $36,9 \%$ haviam sido abandonados pela família, evidenciando a presença de exposição desses a violência multidimensional de natureza complexa e de disparidades socioeconômicas.

No âmbito nacional, houve um aumento significativo na mortalidade de jovens por homicídios em relação à população geral. No Rio Grande do Sul, entre 2002 e 2012, os percentuais de homicídio para a população total passaram de $18,3 \backslash 100.000$ para 21,9\100.000, enquanto que na população jovem (15-24 anos) esta oscilação foi entre 35,9\100.000 e 42,7\100.000 ${ }^{16}$. Analisando o Brasil, a taxa de homicídios para a população total foi de $27,4 \backslash 100.000$ e para a população jovem foi de 54,5\100.0005. No Estado do Rio Grande do Sul, os adolescentes infratores constituem uma parcela da população que muito mais morre do que mata, sendo apontados como autores de apenas $8 \%$ de todas as ocorrências policiais registradas".

Nesse contexto, buscou-se avaliar a relação entre adolescentes infratores e o seu óbito por homicídio na literatura nacional. Não havendo resultados significativos, partiu-se para a busca na literatura internacional, o que reforça a relevância do presente estudo no contexto contemporâneo brasileiro. Aalsma et al. ${ }^{17}$ realizaram um estudo de coorte retrospectivo de jovens envolvidos no sistema penal juvenil ou adulto com uma amostra de 49.479 adolescentes norte americanos, resultando em 518 mortes. Em seu estudo, o pesquisador associou o grau de envolvimento com o sistema de justiça e a mortalidade. As taxas de mortalidade variaram de 90 a $313 \backslash 100.000$ (esta última encontrada em adolescentes julgados pelo sistema penal adulto), ou seja, quanto mais o sistema se aproxima de um perfil adulto e encarcerador, maior é a associação com a mortalidade, não sendo suficientemente esclarecida a relação causa-efeito. Teplin et al. ${ }^{18}$ elaboraram pesquisa com 1.829 participantes adolescentes infratores norte-americanos em período de observação de 16 anos, verificando que apenas 65 egressos haviam falecido (281\100.000), mas que 90,1\% dos óbitos resultaram de homicídios.

Esses estudos realizados nos Estados Unidos da America apresentaram grandes semelhanças entre as amostras e as variáveis aqui avaliadas, apesar de sistemas penais e sociedades distintas. A diferença foi perceptível ao compararmos os estudos: o presente apontou uma taxa de mortalidade de 9,45/100, enquanto Aalsma et al. ${ }^{17}$ descreveu uma de 0,313/100 e Teplin et al..$^{18}$ uma de $0,281 / 100$, apontando a maior violência e vulnerabilidade a que os egressos brasileiros são submetidos. Nosso estudo foi capaz de estabelecer uma relação entre o número de (re) ingressos e desfecho óbito, isto é, adolescentes com 3 ou mais entradas em unidades de internação apresentaram associação com mortalidade. $\mathrm{O}$ grupo com três ou mais ingressos é composto por 1.619 adolescentes, dos quais $208(12,8 \%)$ vieram a óbito - sendo 157 provocados por homicídio.

Além de associação ao homicídio, estes adolescentes evadidos apresentaram altas taxas de suicídio. O suicídio é "todo caso de morte que resulta direta ou indiretamente de um ato positivo ou ne- 
gativo, praticado pela própria vítima, sabedora de que devia produzir esse resultado"19. A sua ocorrência geralmente envolve uma condição psiquiátrica preditora, aliada a fatores de risco das classes sociais extremas (muito pobres ou muito ricos), faixas etárias extremas (idosos ou jovens) ausência de vínculos afetivos e profissionais, ausência de religião e relações familiares conturbadas ${ }^{20}$.

Ao analisarmos dados do DATASUS ${ }^{16}$, relativos ao período de 2002-2012, observou-se entre jovens de 15 a 24 anos uma taxa de suicídio média de 15,68/100.000, a qual foi aproximadamente 155 vezes menor do que a da população analisada no presente estudo. Fica evidente que as penitenciárias recebem um contingente populacional de alto risco para comportamento suicida: homens, jovens, eventualmente depressivos e com transtornos psicóticos, além de indivíduos usuários de drogas, fazendo com que a taxa de suicídios prisionais efetivamente supere a da população em geral ${ }^{21}$.

No caso dos adolescentes, é necessário ponderar as questões familiares e as redes de apoio. A família representa um importante ambiente de socialização e humanização das pessoas, constituindo uma das matrizes do processo civilizatório. Mais do que responder às transformações, as famílias também são geradoras de novas referências sociais, econômicos e demográficos ${ }^{22}$. Gonçalves Zappe e Dias ${ }^{23}$ apontam que jovens infratores têm frequentemente falhas no seu núcleo familiar desde a formação do vínculo mãe -bebê até a adolescência. Nesse sentido, ao comparar-se adultos e jovens expostos e não expostos a situações de violência, observou-se associação da presença da violência familiar com desfechos mentais desfavoráveis como alcoolismo, depressão e ansiedade ${ }^{24}$, contribuindo para o aumento de risco para suicídios.

Estudo realizado com 143 jovens da FASE de Porto Alegre ${ }^{25}$ revelou a presença de idealização suicida de $17,8 \%$, associado a diversos fatores que compõem o perfil do adolescente infrator, entre eles destacam-se: rede de apoio deficitária, baixa escolaridade, ausência de expectativas para o futuro e uso precoce de drogas. Aqui cabe ressaltar que o jovem infrator convive não só com os dilemas intrínsecos à adolescência, mas também com as dificuldades impostas pelo sistema de correção deficitário. Além disso, esse mesmo estudo aponta que $74,8 \%$ desses adolescentes já vivenciaram a morte de alguém importante, ou seja, a violência é algo presente desde cedo em suas vidas e que isso, associado a outras adversidades na infância, está relacionado à ideação e tentativa suicida.
A pesquisa revelou que os adolescentes internados são predominantemente brancos $(53,7 \%)$, informação que deve ser analisada comparativamente com a população do Rio Grande do Sul. Segundo dados do Instituto Brasileiro de Geografia e Estatística ${ }^{26}$, em 2010, cerca de $80 \%$ da população gaúcha era de cor branca. Indicando que adolescentes brancos apresentam uma menor representação proporcional no sistema penal juvenil, conclusão já encontrada em outros estudos realizados no Estado ${ }^{3}$. Esse dado reforça a vulnerabilidade acentuada de etnias diferentes da branca, constituindo-se objeto relevante a ser considerado na formulação de políticas públicas. Por fim, a mortalidade distribui-se entre eles de forma simétrica, o que reforça a hipótese da desigualdade social como denominador comum dos egressos.

Os adolescentes objeto desta pesquisa são majoritariamente do gênero masculino (90,3\%), sendo detectada associação deste grupo com o desfecho óbito. Tal fato está associado ao modelo cultural brasileiro de masculinidade que traz consigo uma forte carga de violência, transmitida aos meninos desde cedo através da socialização e da sua reprodução incentivada ${ }^{27}$. Este perfil de mortalidade acompanha um modelo internacional que também aponta o gênero masculino e os jovens como os mais vulneráveis ${ }^{28}$.

Neste contexto, o limite da idade penal surge como um tema com o qual os resultados deste estudo podem contribuir. Existem diversos argumentos estimuladores da redução da idade penal que levam parte significativa da sociedade a apoiar essa medida. Pesquisa recente aponta um percentual de $87 \%$ dos brasileiros favoráveis à redução da maioridade penal de 18 para 16 anos $^{29}$. Entre os argumentos a favor dessa modificação destaca-se: a utilização de jovens por grupos criminosos na prática de infrações graves, não sendo devidamente julgados e as falhas na aplicação de medidas socioeducativas ${ }^{30}$.

O presente estudo apresenta um cenário caracterizado pela ampla vulnerabilidade de jovens infratores, contrastando com o baixo poder ofensivo de suas infrações. Esses achados oferecem argumento significativo para a rejeição da proposta de redução maioridade penal, pois a sua possível adoção poderá se reverter na ampliação das taxas de mortalidade entre jovens vulneráveis. Há a necessidade de pontuar sistematicamente a associação dos atos infracionais cometidos por adolescentes com suas características de vulnerabilidade social, essas sim, importantes alvos de políticas públicas para as crianças e adolescentes 
do país. Neste contexto, citamos Vaz e Moreira ${ }^{31}$ : "maioridade penal surge como uma tentativa sedutora e desesperada de uma sociedade que falha em cumprir sua responsabilidade social com a população infanto-juvenil”.

A principal limitação deste estudo foi a falta de informações sobre o adolescente relacionada como sua estrutura familiar, escolaridade e suas circunstâncias após a saída da instituição, pois apesar de políticas voltadas ao apoio aos egressos da FASE, não foi possível obter dados sistemáticos dessa amostra. Soma-se a isso o não acesso aos processos legais relacionados a óbitos e mortes violentas. Em relação aos pontos fortes, ressalta-se a alta completude dos dados e uma série temporal longa.

\section{Conclusões}

O Brasil não possui tradição de fomentar políticas voltadas à infância e à adolescência além da educação formal, prática que passou a ser ensaiada apenas recentemente com o advento do Estatuto da Criança e do Adolescente.

O simples fato de não haver dados expressivos na literatura nacional sobre a mortalidade de adolescentes infratores explicita a necessidade de estudos nesta área. A maior parte da literatura está voltada para o motivo pelo qual o adolescente foi internado e não o seu futuro a partir daquele momento, demonstrando a pequena preocupação com o prognóstico das políticas relacionadas aos adolescentes infratores.

Os resultados apontam para a necessidade de um programa de acompanhamento dos egressos associado à formação de uma rede de apoio social extensa e protetora. No contexto da FASE, as práticas e os costumes associados a violência, constrangimento e interdição de indivíduos em fase de desenvolvimento neuropsicológico devem ser revistos, utilizando outras referências contemporâneas voltadas ao pleno aprimoramento dos jovens. Nota-se que alguns estados, dentre eles o Rio Grande do Sul, já possuem programas de apoio aos egressos prisionais com equipes interdisciplinares que fornecem supervisão psicossocial e jurídica ${ }^{32}$, contudo há muito a ser desenvolvido neste aspecto. Finalizando, este estudo aponta a ineficácia de políticas públicas voltadas a um grupo altamente vulnerável de adolescentes infratores, em sua maioria, envolvidos em crimes de baixo poder ofensivo, condenados a uma alta probabilidade de morte.

\section{Colaboradores}

VM Silva participou da coleta de dados, revisão bibliográfica, análise e redação do artigo. PV Teichmann participou da revisão bibliográfica, redação e revisão do artigo. T Scanlon, JVT Santos e MZ Goldani participaram da concepção, elaboração e coordenação do artigo. 


\section{Referências}

1. Brasil. Lei no 8.069 , de 13 de julho de 1990. Dispõe sobre o Estatuto da Criança e do adolescente, e dá outras providências. Diário Oficial da União 1990; 16 de jun.

2. Brasil. Lei no 12.594 , de 18 de janeiro de 2012. Institui o Sistema Nacional de Atendimento Socioeducativo (Sinase), regulamenta a execução das medidas socioeducativas destinadas a adolescente que pratique ato infracional; e altera as Leis nos 8.069 , de 13 de julho de 1990 (Estatuto da Criança e do Adolescente); 7.560, de 19 de dezembro de 1986, 7.998, de 11 de janeiro de 1990, 5.537, de 21 de novembro de 1968, 8.315, de 23 de dezembro de 1991, 8.706, de 14 de setembro de 1993, os Decretos-Leis nos 4.048 , de 22 de janeiro de 1942, 8.621, de 10 de janeiro de 1946, e a Consolidação das Leis do Trabalho (CLT), aprovada pelo Decreto-Lei no 5.452, de 1o de maio de 1943. Diário Oficial da União 2012; 18 jan.

3. Gonçalves Zappe J, Vieira Ramos N. Perfil de adolescentes privados de liberdade em Santa Maria/RS. Psicol. Soc. 2010; 22(2):365-373.

4. Oliveira CS. Sobrevivendo no inferno: a violência juvenil na contemporaneidade. Porto Alegre: Sulina; 2001.

5. Waiselfisz JJ. Mapa da violência: Homicídios por armas de fogo no Brasil. São Paulo: Instituto Sangari; 2016.

6. Brasil. Lei no 2.848 , de 7 de dezembro de 1940 . Institui o código penal. Diário Oficial da União 1940; 7 dez.

7. Brasil. Lei no 11.343, de 23 de agosto de 2006. Institui o Sistema Nacional de Políticas Públicas sobre Drogas - Sisnad; prescreve medidas para prevenção do uso indevido, atenção e reinserção social de usuários e dependentes de drogas; estabelece normas para repressão à produção não autorizada e ao tráfico ilícito de drogas; define crimes e dá outras providências. Diário Oficial da União 2006; 23 ago.

8. Campos MS, Alvarez MC. Pela metade: implicações do dispositivo médico-criminal da "Nova" Lei de Drogas na cidade de São Paulo. Tempo Soc 2017; 29(2):45-73.

9. Ribeiro LM, Rocha RL, Couto VA. Nas malhas da justiça: uma análise dos dados oficiais de indiciados por drogas em Belo Horizonte (2008-2015). Opin Publica 2017; 23(2):397-428

10. Moura NA, Monteiro AR, Freitas RJ. Adolescentes usuários de drogas (i) lícitas e práticas de violência. Rev enferm UFPE on line 2016; 10(5):1685-1693.

11. Rolnik R. Exclusão territorial e violência. Sao Paulo Perspec 1999; 13(4):100-111.

12. Moreira JD, Guerra AM, Drawin CR. Juvenile Crime and Socio-educational Measures: A Literature Review. Psic.: Teor. e Pesq. 2017; 33:e3337

13. Ramos DO, Daly M, Seidl-de-Moura ML, Nadanovsky $P$. The role of city income inequality, sex ratio and youth mortality rates in the effect of violent victimization on health-risk behaviors in Brazilian adolescents. Soc Sci Med 2017; 181:17-23.

14. Moura LB, Oliveira CD, Vasconcelos AM. Violence and youth in a territory of the Metropolitan Area of Brasília, Brazil: a socio-spatial approach. Cien Saude Colet 2015; 20(11):3395-3405.

15. Silva PA, Lerch Lunardi V, Lerch Lunardi G, Algere S, Souza T. Reporting of violence against children and adolescents in a protective institution in southern Brazil. Invest Educ Enferm 2016; 34(1):152-161.
16. Brasil. Sistema do Departamento de Estatística do Sistema Único de Saúde. Datasus. [acessado 2018 Jun 21]. Disponível em: http://www.datasus.gov.br

17. Aalsma MC, Lau KS, Perkins AJ, Schwartz K, Tu W, Wiehe SE, Monahan P, Rosenman MB. Mortality of youth offenders along a continuum of justice system involvement. Am J Prev Med 2016; 50(3):303-310.

18. Teplin LA, McClelland GM, Abram KM, Mileusnic D. Early violent death among delinquent youth: a prospective longitudinal study. Pediatrics 2005; 115(6):1586-1593.

19. Durkheim E. O Suicídio (1897). Lisboa, São Paulo: Editoral Presença, Martins Fontes; 1973.

20. Bonfim CR, Bezerra AL, Góes AB, Farias JR, Guedes KS, Melquiades ML, Sousa MN. Fatores de risco para o suicídio: um estudo de revisão. Intesa 2015; 9(1):76-81.

21. Sarchiapone M. Suicide in the criminal justice system. In: Wasserman D, editor. Suicide: An Unnecessary Death. Oxford: Oxford University Press; 2016. p. 173-177.

22. Goldani AM. As famílias no Brasil contemporâneo e o mito da desestruturação. Cad Pagu 2005; (1):67-110

23. Zappe JG, Dias ACG. Violência e fragilidades nas relações familiares: refletindo sobre a situação de adolescentes em conflito com a lei. Estud Psicol 2012; 17(3):389-395

24. Jaen-Varas D, Jesus MJ, Coutinho ES, Andreoli SB, Quintana MI, Mello MF, Bressan RA, Ribeiro WS. A cross-sectional study to compare levels of psychiatric morbidity between young people and adults exposed to violence in a large urban center. BMC Psychiatry 2016; 16(1):134-142.

25. Nardi FL, Jahn GM, Dell'Aglio DD. Perfil de adolescentes em privação de liberdade: eventos estressores, uso de drogas e expectativas de futuro. Psicologia em Revista 2014; 20(1):116-137.

26. Instituto Brasileiro de Geografia e Estatística (IBGE). Censo Demográfico 2010. Rio de Janeiro: IBGE; 2011. [acessado 2018 Jun 21]. Disponível em: http://www. censo2010.ibge.gov.br/.

27. Regina L, Tognetta P. Educação dos sentimentos: um caminho para a paz. Revista de educação do COGEIME 2005;14(27):23-32.

28. Farrington DP. Early predictors of adolescent aggression and adult violence. Violence Vict 1989; 4(2):79100.

29. Instituto Datafolha. Maioridade Penal. São Paulo: Instituto Datafolha, CNB, CUT; 2016.

30. Barbosa A, Costa GC, Malinoski G, Alves NP. Maioridade Penal No Brasil. JICEX 2017; 5(5).

31. Vaz BG, Moreira J. Responsabilização x Responsabilidades: $\mathrm{o}$ adolescente autor de ato infracional e a redução da maioridade penal. Psicol. Argum. 2017; 33(82):346-363.

32. Oliveira LA, Carvalho MH. O Atendimento ao Adolescente Infrator no Programa de Prestação de Serviço à Comunidade do Município de Matipó. Anais do Seminário Científico da FACIG; 2017 Nov 17-18; Rio de Janeiro.

Artigo apresentado em 06/03/2018

Aprovado em 08/12/2018

Versão final apresentada em 10/12/2018 
